\title{
Study of adhesion between microspheres and rubber surfaces accompanied by meniscus formation and sedimentation
}

\author{
Shoko Mishima, Hiroaki likura and Toshiaki Ougizawa*
}

${ }^{*}$ Correspondence:

tougizawa@op.titech.ac.jp

Graduate School of Science

and Engineering, Tokyo

Institute of Technology,

2-12-1 Ookayama,

Meguro-ku, Tokyo 152-8552,

Japan

\begin{abstract}
This paper reports on the adhesion characteristics between microspheres and rubber surfaces. Silica, polystyrene, and poly(methyl methacrylate) microspheres were deposited on cis-1,4-polybutadiene (BR) films. A BR meniscus formed on the sphere surfaces when the film thickness was less than the diameters of the spheres. Additionally, the attractive forces acting on the spheres in the direction of the BR films were examined via atomic force microscopy. Sedimentation of the spheres occurred for films with thicknesses much greater than the diameters of the microspheres in all systems. Interestingly, this wetting process occurred even in the silica/BR system, despite the incompatibility of these materials. The driving force for meniscus formation is the difference between the surface free energy of $B R\left(\gamma_{B R}\right)$ and that of the spheres $\left(\gamma_{\text {sphere }}\right)$. For all systems, $\gamma_{\mathrm{BR}}$ is lower than $\gamma_{\text {sphere, }}$ i.e., the BR surface is more stable than those of the spheres, and thus a meniscus forms to stabilize the system. Once a meniscus formed, a downward force acted on the spheres to embed them into the BR film. Sedimentation eventually ceased when the angle between the tangential line of the sphere and the rubber surface became equal to the equilibrium contact angle determined by Young's equation. Interestingly, the sedimentation behavior was nearly identical for spheres with various surface free energy values except in terms of their final positions. The same sedimentation phenomena were studied with crosslinked BR films. In contrast to the experiments performed using various types of spheres, the sedimentation behavior varied with different rubber characteristics. The results of these studies indicate that the sedimentation behavior mainly depends on the physical properties of the rubbers used, although the physical properties of the spheres are in determining their final depth.
\end{abstract}

Keywords: Adhesion, Interface, Surface free energy, Meniscus, Sedimentation

\section{Background}

Pressure-sensitive adhesive (PSA) is a common means of adhesion used in various fields. Examples of daily use include adhesive tape or sticky paper. PSAs are also used in industrial fields such as building materials and cars. However, the phenomenon of adhesion is complex because of the many factors involved, such as surface free energy, viscoelasticity, and cohesion. Many studies have been conducted to investigate the nature

(c) The Author(s) 2017. This article is distributed under the terms of the Creative Commons Attribution 4.0 International License (http://creativecommons.org/licenses/by/4.0/), which permits unrestricted use, distribution, and reproduction in any medium, provided you give appropriate credit to the original author(s) and the source, provide a link to the Creative Commons license, and indicate if changes were made. 
of adhesion, most of which have focused on the various adhesion properties between spheres and substrates.

One of the most important aspects involved is the adhesive force. In particular, many theories have been proposed to address deformation induced by the adhesion of spheres on substrates. For example, Johnson et al. [1] investigated the deformation of macroscopic gelatin spheres and presented a theory known as the Johnson-Kendall-Roberts (JKR) theory, which takes a thermodynamic approach to adhesion. An adhesion energy between a rubber ball and a flat smooth glass surface was investigated by Roberts et al. [2]. They observed the time-dependence of contact radius and surface energy with the equation based on JKR theory. These values eventually reached the certain values. The detail of time-dependence of fracture and adhesion energy was investigated by Presson et al. [3]. Furthermore, Derjaguin et al. [4] proposed the Derjaguin-Muller-Toporov (DMT) theory, which includes long-range forces. The JKR theory and the DMT theory each have a certain range of validity [5]. In addition to these theories, the Maugis-Pollock (MP) theory was presented by Maugis et al. [6]. In comparison with the JKR and DMT theories, which assume purely elastic deformation, the MP theory is unique in addressing plastic deformation. High degrees of deformation in substrates have also been studied, for example in a paper by Rimai et al. using a plasticized polystyrene substrate and soda-lime glass spheres [7-9]. Under these conditions, Rimai et al. observed sedimentation of the spheres into the substrate and extended the JKR, DMT, and MP theories to include an understanding of the substrate deformation.

Aside from deformation studies, systems of spheres and substrates have also been investigated to determine the fundamental physical properties of polymers. For instance, Sharp et al. [10] directly assessed the near-surface properties of polystyrene films by monitoring how gold nanoparticles became embedded. The adhesive force between spheres and substrates coated with low viscosity liquids, such as water or silicon oil, have been investigated using a capillary bridge with atomic force microscopy (AFM) [11-14]. The measured force has been fitted to a hydrodynamic force, van der Waals attraction, electrostatic repulsion, hydrodynamic drag, and the restoring force of a cantilever [11, 13]. In addition to these forces, Ally et al. [14] has taken capillary forces into account, which has been a topic of interest among researchers for many years. Cross [15] and Pietsch [16] and their coworkers showed that the capillary force component results from the direct action of the surface free energy, with further details recently reported by Butt et al. [17].

In contrast to studies of deformation and adhesive force performed on low viscosity materials, few reports on the initiation of the adhesion process and wetting progress in viscoelastic materials are available. Therefore, the relationship between the initial stage of stickiness and the physical properties of polymers is not well understood.

In this study, we have investigated the adhesion characteristics between micro-scale spheres and rubber films with large thicknesses relative to the sphere diameters. Viscoelastic materials such as rubber generally require a long time to become wetted. To address this problem, we used spheres with sizes on the order of microns. For such small spheres, wetting at the surface proceeds in a sufficiently short time that we were able to observe the process. Additionally, we were able to observe deformation of the viscoelastic material caused by wetting. We focused on four main factors in order to investigate 
the process of adhesion, including two physical properties of the spheres, the surface free energy and the sphere size, in addition to the surface free energy, and viscoelasticity of rubber.

\section{Experimental Materials}

cis-1,4-Polybutadiene (BR) (BR01) was supplied by JSR Corp. The glass transition temperature $T_{\mathrm{g}}$ of this material is $-108^{\circ} \mathrm{C}$, while the number average of the molecular weight $M_{\mathrm{n}}$ is 121,193 and $M_{\mathrm{w}} / M_{\mathrm{n}}$ is 4.36 . Dicumyl peroxide (DCP), which is a crosslinking agent for BR, was purchased from Sigma-Aldrich, Inc. Three types of silica spheres (Hyprecia) were obtained from Ube-Exsymo Co., Ltd with diameters of 5, 10, and $50 \mu \mathrm{m}$. Polystyrene (PS) spheres (SBX-12) and poly(methyl methacrylate) (PMMA) spheres (MB-8) were supplied by Sekisui Plastics Co., Ltd with diameters of 12 and $8 \mu \mathrm{m}$, respectively.

\section{Rubber film preparation}

Rubber films were prepared with two thicknesses. To prepare thin BR films with thicknesses around $500 \mathrm{~nm}$, a BR solution in toluene (3.0 wt\%) was spin-coated at $2000 \mathrm{rpm}$ for $30 \mathrm{~s}$ onto a single-crystal silicon wafer with a native oxide layer (Mitsubishi Materials Trading Corp.). The film was dried for at least $24 \mathrm{~h}$ at room temperature and the film thickness was evaluated with an ellipsometer (SA-101; Photonic Lattice, Inc.).

Rubber films with large thicknesses compared to the diameter of the spheres were prepared by a solvent cast method. A BR solution in toluene (10 wt\%) was cast onto a silicon wafer and the resulting film was dried for $24 \mathrm{~h}$ at room temperature in ambient conditions and for $24 \mathrm{~h}$ under vacuum. The film thickness was determined to be around $160 \mu \mathrm{m}$ by scanning electron microscopy (SEM) (SM-200; Topcon Corp.). For thicker films, a crosslinked BR film was also made using a BR solution in toluene (10 wt\%; DCP $1.5 \mathrm{phr}$ for rubber). After drying in ambient conditions followed by vacuum drying, the film was annealed at $130{ }^{\circ} \mathrm{C}$ for $0,15,20,25$, and 30 min to induce crosslinking. Hereafter, the crosslinked samples are denote by the crosslinking time appended to BR (e.g., BR15 describes a BR film crosslinked for $15 \mathrm{~min}$ ).

The surface free energies of the rubber films were evaluated with the Owens-Wendt equation [18]. Because two types of contact angles are required for this evaluation, we used ultrapure water and diiodomethane, and determined the contact angles with a Dropmaster 300 from Kyowa Interface Science Co., Ltd.

The storage modulus $G^{\prime}$ and the loss modulus $G^{\prime \prime}$ of the rubber were measured with a rheometer (parallel plate, $\phi=25 \mathrm{~mm}, 20{ }^{\circ} \mathrm{C}$, Rheometrics dynamic mechanical spectrometer RDS-7700; Rheometrics, Inc.).

The shear moduli of the rubber films were evaluated from the force-distance curve measured by atomic force microscopy (AFM) (MFP-3D-BIO-J; Asylum Research) and the Johnson-Kenall-Roberts (JKR) theory [1] was applied to the evaluation. The JKR theory is known to be suitable for highly adhesive systems with low stiffness and large tip radii, and can therefore be applied to the system in this study. In several reports on this theory $[19,20]$, the "2-point method" has been proposed for determining the elastic modulus, $E$. The shear modulus, $G$, can then be calculated using the following equation: 


$$
G=\frac{E}{2(1+v)}=\frac{E}{3}
$$

where $v$ is the Poisson's ratio, which was assumed to be 0.5 in this study. The value of 0.5 is a typical Poisson's ratio for a non-compressive elastomer.

\section{Test for adhesion of spheres to rubber surfaces}

The spheres were deposited onto the rubber films, then nitrogen gas was blown across the rubber surfaces to remove any unattached spheres. The interfacial area between the spheres and the rubber was imaged using SEM after resting the samples for a designated length of time.

\section{Force-distance curves from particle probe measurements}

The adhesion between the silica spheres and thin rubber films was investigated by AFM. These measurements used a silicon cantilever with a silica sphere at the tip $(23.9 \mathrm{~N} / \mathrm{m}$ spring constant, $10 \mu \mathrm{m}$ silica sphere diameter, $400 \mathrm{~nm} / \mathrm{s}$ scanning rate, Novascan Technologies, Inc.).

The attractive force was also measured by AFM by first moving the sample stage to approach the cantilever and pausing the approach after contact occurred between the substrate and the cantilever following a jump in the force curve. Then, the measured force returned to $0 \mathrm{~N}$, the sample stage was fixed, and the changes in detected force with time were recorded.

\section{Equilibrium contact angle measurements}

A 500-nm-thick BR film was produced using the above method. The film was scratched with tweezers, and submicron fragments of BR that remained attached to the tip of the tweezers were placed on a flat silica glass surface. Changes in the contact angle with time were observed by SEM.

\section{Results and discussion}

Figure 1 shows an SEM image of the interface between a silica sphere with a $10 \mu \mathrm{m}$ diameter and a BR film with a $500 \mathrm{~nm}$ thickness. The image clearly illustrates the formation of a BR meniscus on the silica sphere. Adhesion of the silica occurred quickly, in $<10 \mathrm{~s}$, so the rubber meniscus must have formed immediately after the spheres were deposited. Generally, contact between hydrophilic and hydrophobic materials tends to result in smaller contact areas; yet, the hydrophobic BR formed a meniscus on the hydrophilic silica surface. This phenomenon can be understood in terms of the differences in surface free energy. The surface free energy of BR is $28.8 \mathrm{~mJ} / \mathrm{m}^{2}$ and that of silica is $>300 \mathrm{~mJ} / \mathrm{m}^{2}$. In other words, a BR/air system is more stable than a silica/air system, so the BR tends to cover the silica sphere surface. As a result, a BR meniscus formed on the silica sphere surface even though they are not compatible. In addition to silica, the surface free energies of PS and PMMA are 43 and $39 \mathrm{~mJ} / \mathrm{m}^{2}$, respectively, which are both larger than that of BR, also resulting in the formation of a BR meniscus on PS and PMMA sphere surfaces. 


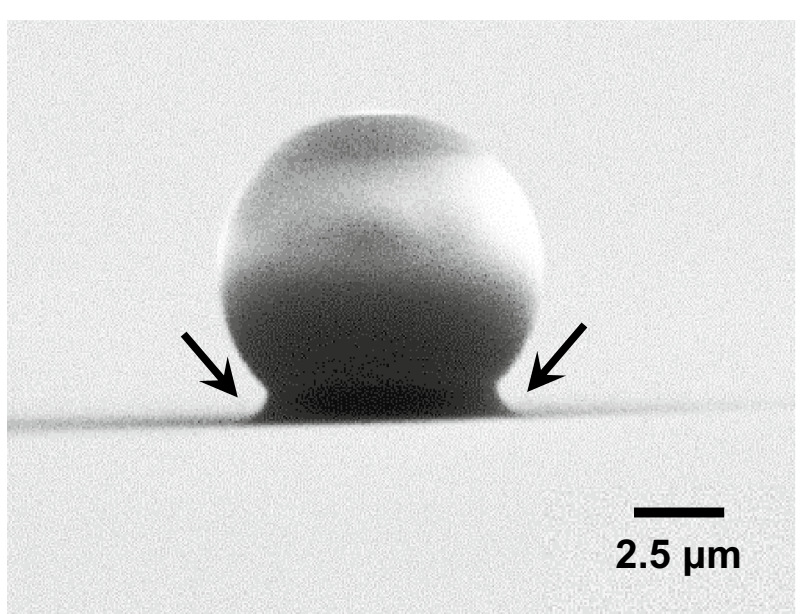

Fig. 1 SEM image of the interface between a thin BR film and a silica sphere. $10 \mu \mathrm{m}$ sphere diameter; $500 \mathrm{~nm}$ BR film thickness

Figure 2a displays the force-distance curve for a thin BR film (500 nm thickness) measured by a cantilever with a silica sphere tip. As shown in Fig. 2a, the cantilever first adhered to the BR film by van der Waals forces (point A in Fig. 2a); then, the cantilever returned to a straight position by lifting the sample stage (from point $\mathrm{B}$ to $\mathrm{C}$ ) until the force decreased to zero. Afterward, the sample stage was fixed at point $C$. The force curves measured after fixing the sample stage are shown in Fig. 2b. The data plot indicates that downward forces were detected and the cantilever gradually bent, reflecting an attractive force on the silica sphere as a result of the meniscus (Fig. 3), known as the meniscus force, $F_{\text {meniscus }}$.

The meniscus force consists of two components [17]:

$$
F_{\text {meniscus }}=F_{\text {Lap }}+F_{\text {cap }}
$$

the Laplace force, $F_{\mathrm{Lap}}$, and the capillary force, $F_{\text {cap }}$, which is induced by the Laplace pressure, $\Delta p$. This pressure is generated in the vicinity of the interface between two fluids
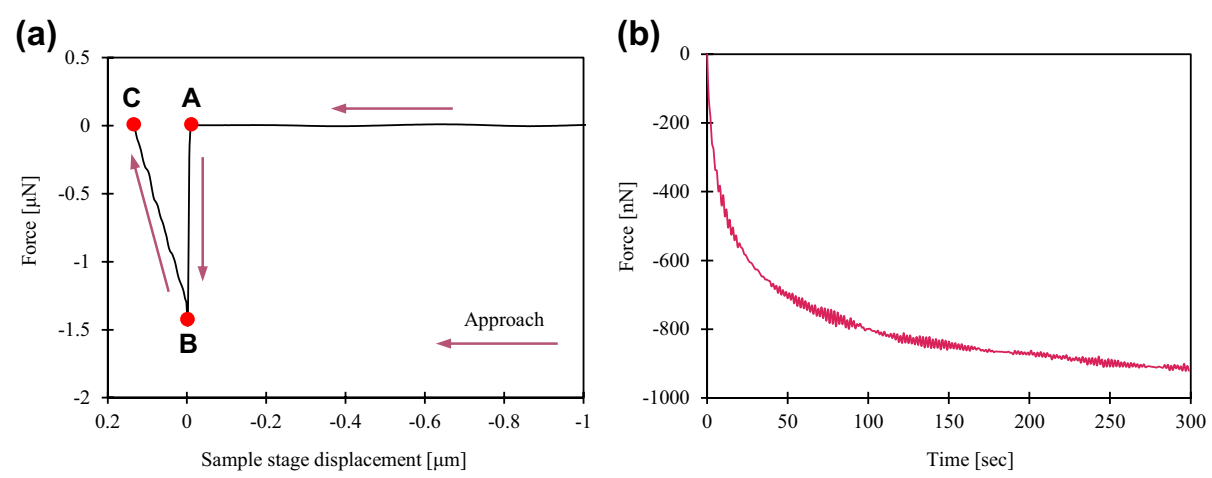

Fig. 2 AFM measurement of a thin BR film using a cantilever attached to a silica sphere tip. a Force-distance curve of a thin BR film. b Variation in the attractive force with time. Conditions: $500 \mathrm{~nm}$ BR film thickness; $10 \mu \mathrm{m}$ silica sphere diameter; $23.9 \mathrm{~N} / \mathrm{m}$ cantilever spring constant; $400 \mathrm{~nm} / \mathrm{s}$ scanning rate 


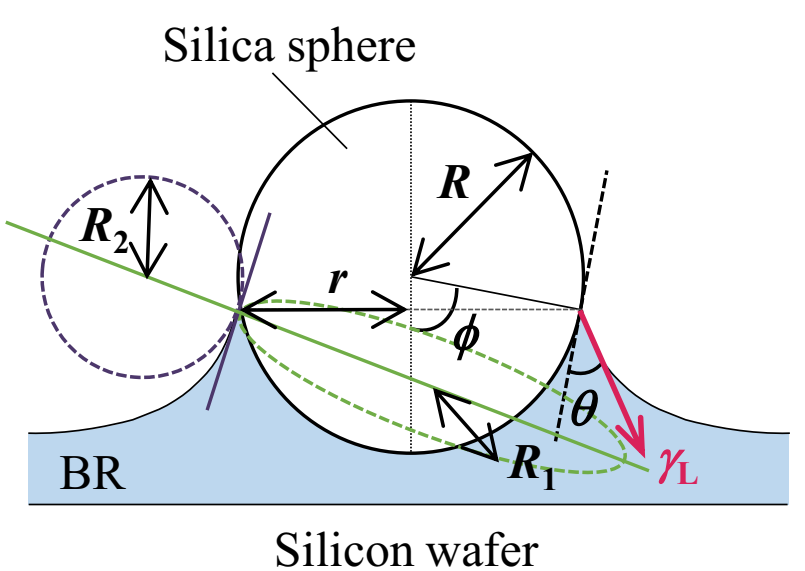

Fig. 3 Schematic of a meniscus between a sphere and a flat substrate

that have different surface free energies. $F_{\text {Lap }}$ is described by the following equations [17, 21, 22]:

$$
\begin{aligned}
& F_{\text {Lap }}=S \Delta p=\pi r^{2} \Delta p \\
& \Delta p=\gamma_{\mathrm{L}}\left(\frac{1}{R_{1}}+\frac{1}{R_{2}}\right)\left(R_{1}>0, R_{2}<0\right)
\end{aligned}
$$

where $S$ is the contact area, $\left(1 / R_{1}+1 / R_{2}\right)$ is the surface curvature of an interface, and $\gamma_{\mathrm{L}}$ is the surface free energy of the liquid.

The $F_{\text {cap }}$ portion of $F_{\text {meniscus }}$ is induced by $\gamma_{\mathrm{L}}$ at the contact line of a meniscus [14, 17, 23, 24]:

$$
F_{\text {cap }}=l \gamma_{L} \sin (\phi+\theta)=2 \pi r \gamma_{L} \sin (\phi+\theta)
$$

where $l$ is the length of the contact line, and the angles $\phi$ and $\theta$ are shown in Fig. 3. The direction of $\gamma_{\mathrm{L}}$ is tangential to the line of liquid around the contact line. These forces produced an attractive force that acted on the sphere at the tip of the AFM cantilever. The meniscus of rubber formed immediately on the sphere surface after point $B$, then the meniscus force acted on the sphere and the sphere became embedded in the BR film. As a result, the cantilever was bent and a force was detected (Fig. 2b).

Because the attractive force acts on the spheres in the direction of the rubber film, we used thicker rubber films in place of the thin film to investigate the wetting process. These conditions resulted in sedimentation of the silica spheres into the rubber film. Figure 4 shows SEM images of this sedimentation, as well as similar sedimentation features for PS spheres and PMMA spheres. The sedimentation ratio, $\delta / D$, was calculated from SEM images to compare the sedimentation behavior of each sphere type, where the term $\delta$ is the sedimentation depth and $D$ is the diameter of the spheres. Figure 5 shows the time-dependence of sedimentation for all three types of spheres. According to Fig. 5, the sedimentation behavior indicated by the shape of the curves was nearly identical for all spheres. Additionally, the sedimentation velocities are also similar for all spheres, even for spheres with very different surface free energies. In comparison to the silica/air 

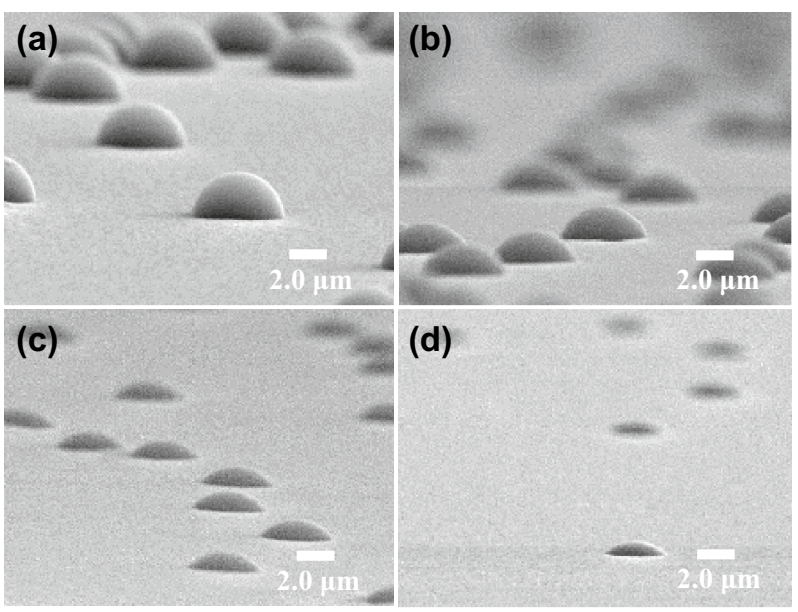

(d) diameter; resting times: a $5 \mathrm{~min}, \mathbf{b} 30 \mathrm{~min}, \mathbf{c} 10 \mathrm{~h}$, and $\mathbf{d} 792 \mathrm{~h}$

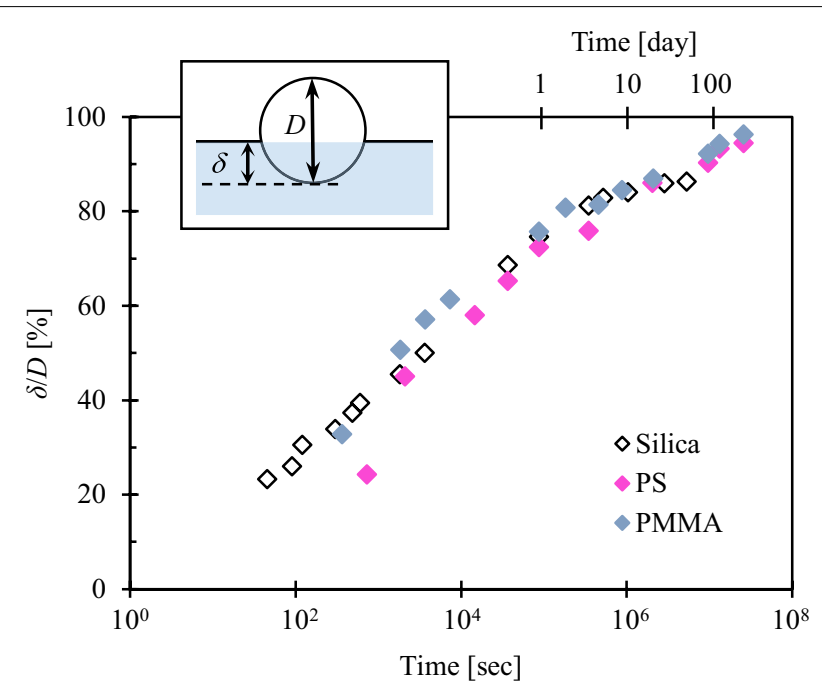

Fig. 5 Resting-time-dependence of sedimentation ratios for silica, PS, and PMMA spheres in a thick BR film. Sphere diameters: silica $10 \mu \mathrm{m}$, PS $12 \mu \mathrm{m}$, PMMA $8 \mu \mathrm{m}$; BR film thickness: $160 \mu \mathrm{m}$

system, the polymer/air systems are more stable as a result of their smaller surface free energy. Therefore, the silica spheres were expected to be more rapidly embedded into the rubber film than the polymeric spheres, however a different result was obtained. Figure 5 indicates that the sedimentation behavior does not depend strongly on the physical properties of the spheres. These results are also in agreement with Eqs. (4) and (5), which indicate that the attractive force depends only on the physical properties of the rubber. Additionally, we assume a drag force occurs by the viscoelastic deformation of rubber. We discuss the dependence of viscoelasticity of rubber later.

Figure 5 also shows that sedimentation eventually ceased at a certain depth, depending on the type of sphere. The final depths were $85 \%$ for silica spheres, $95 \%$ for PS spheres, and 96\% for PMMA spheres. The angle between the rubber surface and the tangential 
line to the sphere at its final position, $\theta_{\text {end }}$, was calculated using the final depth, $\delta / D$. These values were $45^{\circ}, 26^{\circ}$, and $23^{\circ}$, respectively. According to Eqs. (4) and (5), the sedimentation should cease when the meniscus has vanished. Disappearance of the meniscus requires that $\Delta p=0$ and $\theta=\pi-\phi$, then $F_{\text {Lap }}=0$ and $F_{\text {cap }}=0$.

Next, we considered the factors that determine the final sedimentation position of the spheres. The terminal contact angle is assumed to be equal to the equilibrium contact angle, $\theta_{\text {eq }}$ in Young's equation [25]:

$$
\gamma_{\mathrm{S}}=\gamma_{\mathrm{SL}}+\gamma_{\mathrm{L}} \cos \theta_{\mathrm{eq}}
$$

Here, $\gamma_{\mathrm{S}}$ and $\gamma_{\mathrm{L}}$ are the surface free energy of the solid and liquid, respectively, and $\gamma_{\mathrm{SL}}$ is the interfacial free energy between the liquid and the solid. For a sphere at the liquidgas interface, the sphere is stable at the position where the forces on the tangential line around the contact line are balanced [10,26]. Namely, in equilibrium, Young's equation is valid for the tangential line to the sphere. To verify whether this theory also holds in our system, in which spheres are present at a viscoelastic material-gas interface, we determined $\theta_{\text {eq }}$ by the following experiment and compared the values of $\theta_{\text {eq }}$ with $\theta_{\text {end }}$. Pieces of BR with an equilibrium contact area diameter of around $30 \mu \mathrm{m}$ were placed on silica glass substrates. The time-dependence of the contact angle is shown in Fig. 6. The BR pieces gradually spread along the surface of the silica glass. The final contact angle reached a value of $43^{\circ}$, which is broadly consistent with the $\theta_{\text {end }}$ value of $45^{\circ}$. This result indicates that a sphere at a viscoelastic material-gas interface, as well as the liquid-gas system, is in equilibrium when $\theta_{\text {end }}$ becomes equal to $\theta_{\text {eq }}$. We also confirmed whether the value of $\theta_{\text {eq }}$ was appropriate comparing the apparent surface free energy reported by Roberts et al. [2]. We calculated the interfacial free energy using Young's equation [Eq. (6)], and it was about $278 \mathrm{~mJ} / \mathrm{m}^{2}$. Then, we also calculated the work of adhesion, $w$, using Dupré equation [21]:

$$
\gamma_{\mathrm{SL}}=\gamma_{\mathrm{S}}+\gamma_{\mathrm{L}}-w
$$

$w$ between the BR and the glass substrate was about $51 \mathrm{~mJ} / \mathrm{m}^{2}$. Robert and his coworker reported $w$ of natural rubber (NR)-glass system was $60 \mathrm{~mJ} / \mathrm{m}^{2}$. BR and NR are

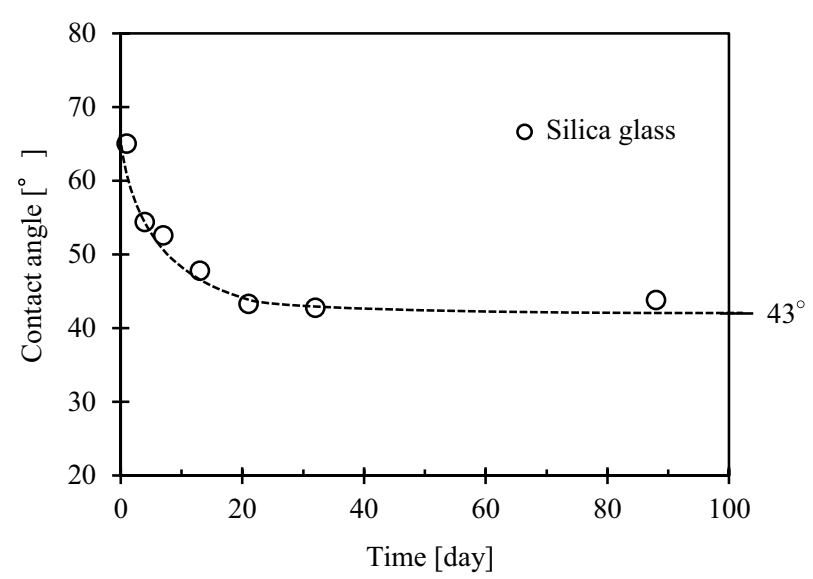

Fig. 6 Resting-time-dependence of contact angle between BR pieces and silica glass 
considered to have similar adhesion properties, therefore our $w$ value is considered to be an appropriate value. Additionally, our experiments show new method to obtain an interfacial free energy between a solid and a viscoelastic material directly using Young's equation by just placing the micron-order viscoelastic piece on the solid.

\section{Dependence on sphere size}

To confirm the influence of sphere size on the sedimentation behavior, various sizes of silica spheres were deposited onto a thick BR film without a crosslinking agent. Figure 7a shows the time-dependence of sedimentation. The velocities clearly varied among the three spheres sizes, however, the final sedimentation ratios had very similar values. The plots in Fig. 7a, with a reference sphere size of $D=50 \mu \mathrm{m}$, can be superposed on one another by shifting the data along the time axis (Fig. 7b). Figure 8 displays a log$\log$ plot of the relationship between the shift factors, $a_{t}$, and the sphere diameter, which

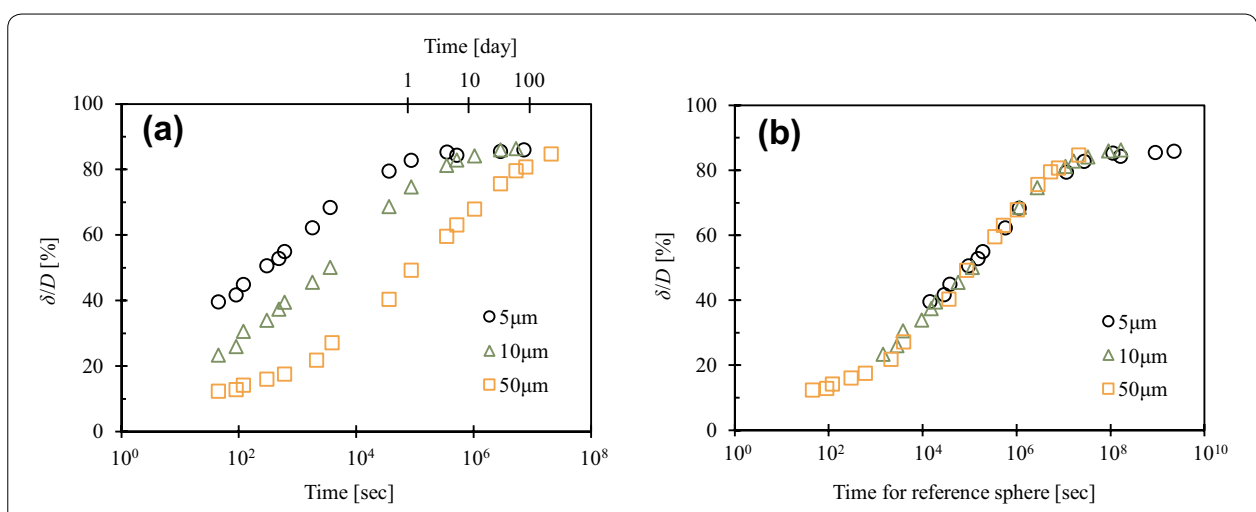

Fig. 7 Resting-time-dependence of sedimentation ratios of silica spheres with various diameters in a thick BR film. a Raw data, b data shifted along the time axis. The reference sphere size is $50 \mu \mathrm{m}$

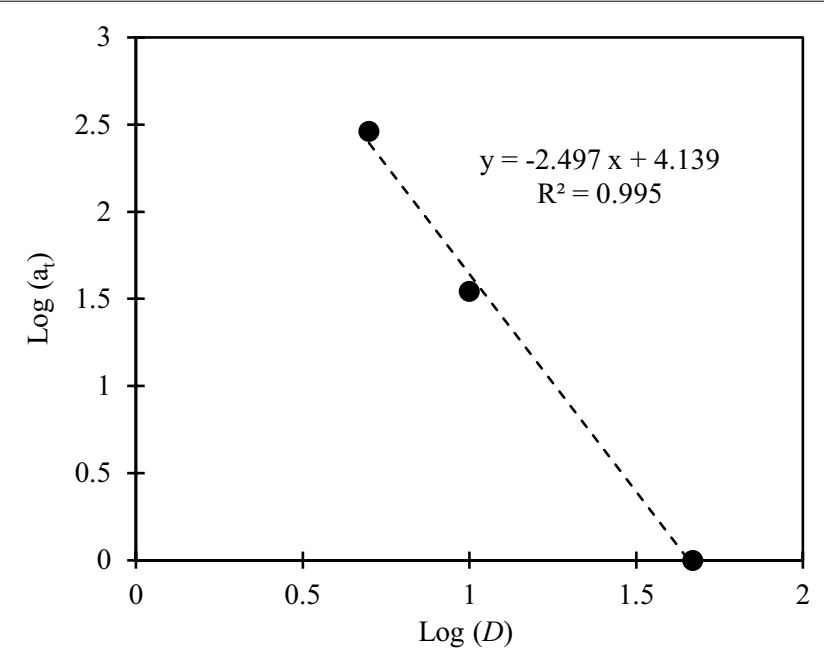

Fig. 8 Shift factors for plotted data in Fig. 7b versus sphere diameter. The dotted line represents a linear relationship 
approximates a straight line. The slope of 2.5 for this line indicates that the total time for sedimentation is proportional to $D$ to the power of 2.5. This result clearly shows the relaxation process is dominated by time-dependent fracture energy, therefore we have to clarify the relationship between $D$ and $\mathrm{a}_{\mathrm{t}}$ in the near future. In contrast to the velocity, the sedimentation behavior does not depend on sphere size (i.e., the curve shapes were nearly identical), as the shape of all plots was consistent. As described above, the final depth depends only on the physical properties of the spheres and the rubber: $\gamma_{S}, \gamma_{L}$, and $\gamma_{\mathrm{SL}}$. The sphere size affects the velocity but not the overall behavior.

\section{Dependence on rubber type}

As discussed above, the type of sphere does not affect the intermediate stage of sedimentation behavior, however the final sphere depth depends significantly on the material. Therefore, we considered the effect of different types of rubber. For this investigation, we observed the sedimentation behavior of silica spheres into crosslinked BR films. Figure 9 shows the cross-linking time-dependence of the storage modulus $G^{\prime}$ and the loss modulus $G^{\prime \prime}$. This result indicates that the fluidity of the BR film decreases with crosslinking time. Figure 10 shows the cross-linking time-dependence of the surface free energy (Fig. 10a) and the shear modulus (Fig. 10b). Both values increased with

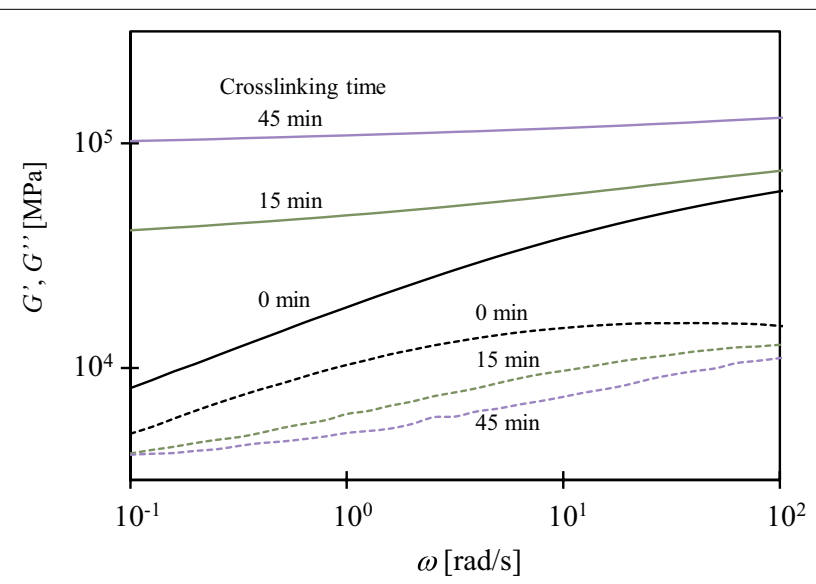

Fig. 9 Cross-linking time-dependence of the storage modulus $G^{\prime}$ (solid lines) and the loss modulus $G^{\prime \prime}$ (dashed lines) for a thick BR film
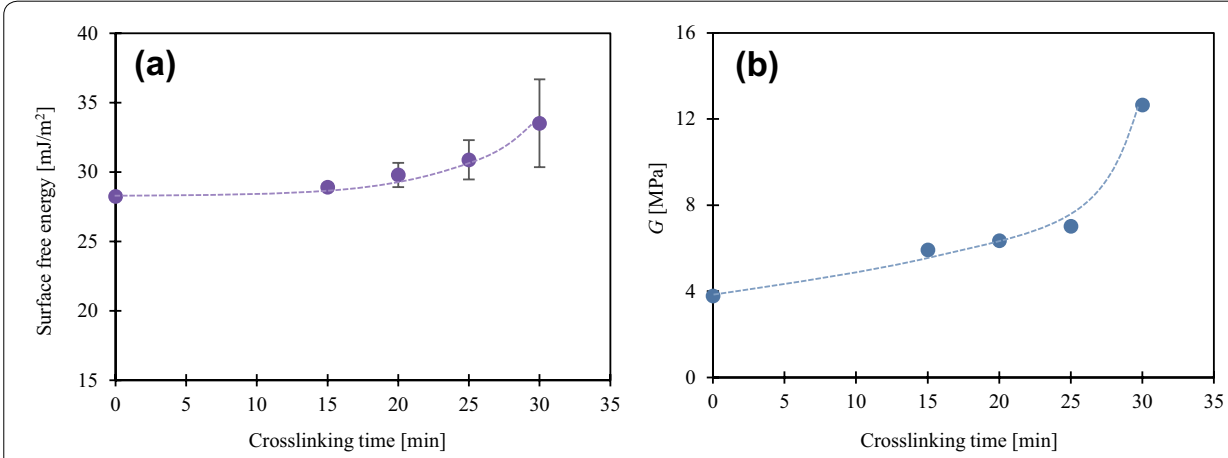

Fig. 10 Cross-linking time-dependence of $\mathbf{a}$ surface free energy and $\mathbf{b}$ shear modulus $G$ for a thick BR film 
greater crosslinking times. The reason for increasing of the surface free energy as the crosslinking proceeds is increases of a cohesive force and a density of the BR. To study the sedimentation behavior, silica spheres with a diameter of $5 \mu \mathrm{m}$ were deposited on BR0, BR15, and BR30 films. The results are shown in Fig. 11a. The plots for BR15 and BR30 were shifted to compare the sedimentation behavior of these samples with that of BR0 (Fig. 11b). Figure 11b demonstrates that the shapes of the curves are very similar, whereas the final sphere depth for BR30 is different from that of the other samples. Details are provided below.

First, the surface free energy of BR0 is nearly the same as that of BR15 according to Fig. 10a; however, the shear modulus increase with crosslinking time. Therefore, the flow of BR15 from the bottom to the side of a sphere and the sedimentation of BR15 were slower than that of BR0. This result suggests that the sedimentation velocity relies on the viscoelasticity of the rubber film. Secondly, BR30 clearly exhibited a different terminal sedimentation position than the other two samples. Because the surface free energy of BR30 is larger than those of BR0 and BR15, the positions predicted by Young's equation and thus the final depths were different. These results indicate that the sedimentation mechanism is strongly influenced by the physical properties of the rubber film, such as surface free energy and viscoelasticity. These results are also in agreement with Eqs. (4) and (5), which indicate that the attractive force depends only on the physical properties of the rubber.

\section{Conclusions}

We have reported the adhesion characteristics between microspheres and rubber surfaces. For thin rubber films with thicknesses less than the sphere diameters, the formation of a rubber meniscus was observed on the sphere surfaces. In the case of thick rubber films, with large thicknesses compared to the sphere diameters, sedimentation of the microspheres occurred. These studies demonstrate certain important features about the sedimentation mechanisms:

- A meniscus formed immediately on the sphere surfaces after they were deposited. This result indicates that an attractive force develops in a short time span $(<10 \mathrm{~s})$.

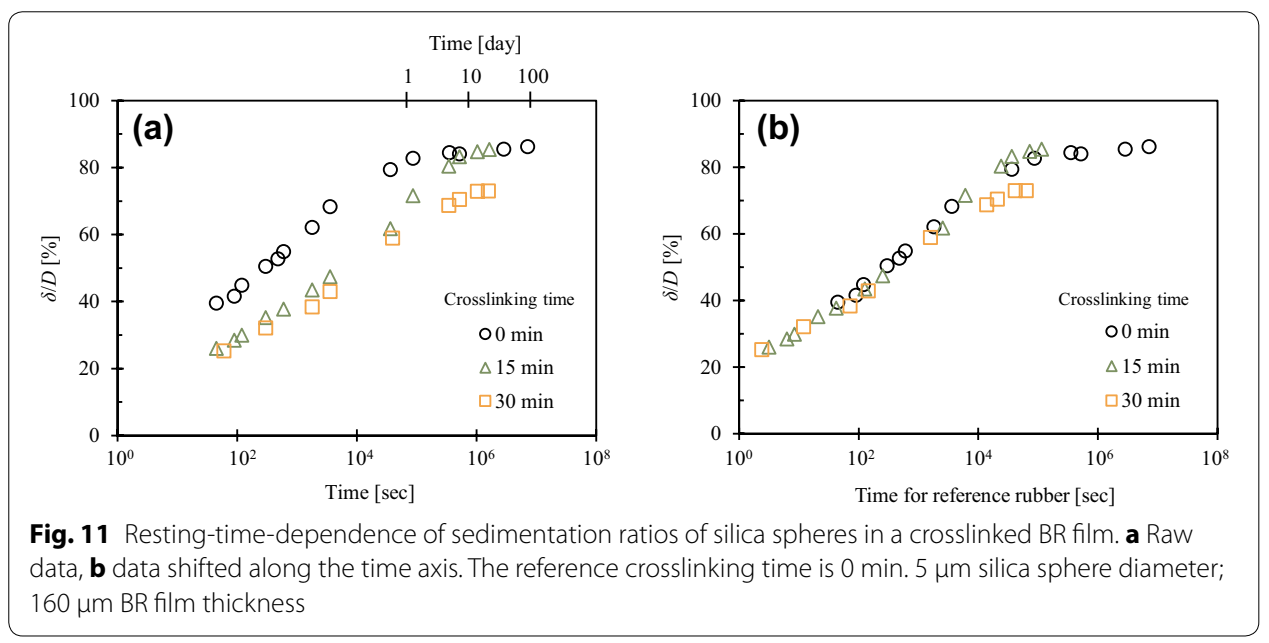


- The system was stabilized as a result of meniscus formation and microspheres becoming embedded in the rubber film.

- Young's equation is valid to describe the final sphere depth. In other words, the angle between the rubber surface and the tangential line of the sphere in its final position, $\theta_{\text {end }}$, is equal to the equilibrium contact angle, $\theta_{\text {eq }}$, in Young's equation.

The following relationships between the sedimentation phenomena and material factors were also determined:

- Sedimentation behavior mainly depends on the physical properties of the rubber.

- Sedimentation behavior is nearly independent of the physical properties of the spheres.

- The physical properties of the spheres are particularly important in determining their final depth.

\section{Abbreviations}

BR: cis-1,4-polybutadiene; NR: natural rubber; PSA: pressure-sensitive adhesive; JKR: Johnson-Kendall-Roberts; DMT: Derjaguin-Muller-Toporov; MP: Maugis-Pollock; AFM: atomic force microscopy; DCP: dicumyl peroxide; PS: polystyrene; PMMA: poly(methyl methacrylate); SEM: scanning electron microscopy; $T_{\mathrm{g}}$ : glass transition temperature; $M_{\mathrm{n}}$ : number average of the molecular weight; $M_{w}$ : weight average of the molecular weight; $M_{w} / M_{n}$ : molecular weight distribution; $G^{\prime}$ : storage modulus; $G$ ": loss modulus; $E$ : elastic modulus; $G$ : shear modulus; $v$ : Poisson's ratio; $F_{\text {meniscus: }}$ meniscus force; $F_{\text {Lap: }}$ : Laplace force; $F_{\text {cap }}$ : capillary force; $\triangle p$ : Laplace pressure; $S$ : contact area; $R_{1}, R_{2}$ : curvature radii of an interface; $\left(1 / R_{1}+1 / R_{2}\right)$ : surface curvature of an interface; $\gamma_{S}$ : surface free energy of the solid; $\gamma_{\mathrm{L}}:$ surface free energy of the liquid; $\gamma_{\mathrm{SL}}:$ interfacial free energy between the liquid and the solid; $/$ : length of the contact line; $\phi$ : filling angle; $\theta$ : contact angle; $D$ : diameter of the sphere; $\delta$ : sedimentation depth; $\delta / D$ : sedimentation ratio; $\theta_{\text {end: }}$ angle between the rubber surface and the tangential line to the sphere at its final position; $\theta_{\text {eq: }}$ equilibrium contact angle in Young's equation; $w$ : work of adhesion; $a_{t}$ : shift factor.

\section{Authors' contributions}

SM prepared the manuscript, observed sedimentation of PS sphere and PMMA sphere. HI conceived of the study, did observations with system of silica sphere and BR film, and carried out AFM measurement. TO conceived of the study, participated in its design and coordination, and revised the draft manuscript. All authors read and approved the final manuscript.

\section{Acknowledgements}

Not applicable.

\section{Competing interests}

The authors declare that they have no competing interests.

\section{Availability of data and materials}

All materials we used were supplied by the companies which we wrote in the material section of main manuscript.

Received: 1 November 2016 Accepted: 26 January 2017

Published online: 06 February 2017

\section{References}

1. Johnson KL, Kendall K, Roberts AD. Surface energy and the contact of elastic solids. Proc R Soc Lond Ser A. 1971;324(1558):301-13.

2. Roberts AD, Thomas AG. The adhesion and friction of smooth rubber surfaces. Wear. 1975;33:45-64.

3. Persson BNJ, Albohr O, Heinrich G, Ueba H. Crack propagation in rubber-like materials. J Phys: Condens Matter. 2005; 17:R1071-142

4. Derjaguin BV, Muller VM, Toporov YuP. Effect of contact deformations on the adhesion of particles. J Colloid Interface Sci. 1975;53(2):314-26

5. Muller VM, Yushchenko VS, Derjaguin BV. On the influence of molecular forces on the deformation of an elastic sphere and its sticking to a rigid plane. J Colloid Interface Sci. 1980;77(1):91-101.

6. Maugis D, Pollock HM. Surface forces, deformation and adherence at metal microcontacts. Acta Metall. 1984;32(9):1323-34

7. Rimai DS, Quesnel DJ, Busnaina AA. The adhesion of dry particles in the nanometer to micrometer-size range. Colloids Surf. 2000;165(1-3):3-10. 
8. Rimai DS, Schaefer DM, Bowen RC, Quesnel DJ. The time dependence of particle engulfment. Langmuir. 2002;18(12):4592-7.

9. Rimai DS, Ezenyilimba MC, Quesnel DJ. Effects of electrostatic and van der Waals interactions on the adhesion of spherical $7 \mu \mathrm{m}$ particles. J Adhes. 2005;81(3-4):245-69.

10. Sharp JS, Teichroeb JH, Forrest JA. The properties of free polymer surfaces and their influence on the glass transition temperature of thin polystyrene films. Eur Phys J E. 2004;15(4):473-87.

11. Guriyanova S, Bonaccurso E. Influence of wettability and surface charge on the interaction between an aqueous electrolyte solution and a solid surface. Phys Chem Chem Phys. 2008;10:4871-8.

12. Butt H-J, Cappella B, Kappl M. Force measurements with the atomic force microscope: technique, interpretation and applications. Surf Sci Rep. 2005;59(1-6):1-152.

13. Guriyanova S, Semin B, Rodrigues TS, Butt H-J, Bonaccurso E. Hydrodynamic drainage force in a highly confined geometry: role of surface roughness on different length scales. Microfluid Nanofluid. 2010;8(5):653-63.

14. Ally J, Vittorias E, Amirfazli A, Kappl M, Bonaccurso E, McNamee CE, Butt H-J. Interaction of a microsphere with a solid-supported liquid film. Langmuir. 2010;26(14):11797-803.

15. Cross NL, Picknett RG. Particle adhesion in the presence of a liquid film. In: Johnson HR, Littler DJ, editors. International conference on mechanism of corrosion by fuel impurities, Marchwood 1963. Marchwood: Butterworths; 1963. p. 383-90.

16. Pietsch W, Rumpf H. Haftkraft, kaplliardruck, flüssigkeitsvolumen und grenzwinkel einer flüssigkeitsbrücke zwischen zwei kugeln. Chem Ing Technol. 1967;39(15):885-93.

17. Butt H-J, Kappl M. Normal capillary forces. Adv Colloid Interface Sci. 2009;146:48-60.

18. Owens DK, Wendt RC. Estimation of the surface free energy of polymers. J Appl Polym Sci. 1969;13(8):1741-7.

19. Sun Y, Akhremitchev B, Walker GC. Using the adhesive interaction between atomic force microscopy tips and polymer surfaces to measure the elastic modulus of compliant samples. Langmuir. 2004;20(14):5837-45.

20. Nagai S, Fujinami S, Nakajima K, Nishi T. Nanorheological investigation of polymeric surfaces by atomic force microscopy. Compos Interfaces. 2009;16(1):13-25.

21. Israelachvili JN. Intermolecular and surface forces. 2nd ed. London: Academic press; 1992.

22. Matthewson MJ. Adhesion of spheres by thin liquid films. Philos Mag A. 1988;57(2):207-16.

23. Orr FM, Scriven LE. Pendular rings between solids: meniscus properties and capillary force. J Fluid Mech. 1975;67(4):723-42.

24. de Gennes PG, Brochard-Wyart F, Quéré D. Gouttes, bulles, perles et ondes. Paris: Belin; 2002.

25. Young T. An essay on the cohesion of fluids. Philos Trans R Soc. 1805:95:65-87.

26. Butt H-J, Graf K, Kappl M. Physics and chemistry of interfaces. Weinheim: Wiley-VCH Verlag \& Co. KGaA; 2003.

\section{Submit your manuscript to a SpringerOpen ${ }^{\circ}$ journal and benefit from:}

- Convenient online submission

Rigorous peer review

- Immediate publication on acceptance

Open access: articles freely available online

- High visibility within the field

- Retaining the copyright to your article

Submit your next manuscript at $>$ springeropen.com 